\title{
Prosodic focus in three northern Wu dialects: Wuxi, Suzhou and Ningbo
}

\author{
Bei Wang ${ }^{1}$, Yuanyuan Zhang ${ }^{2}$, Yi Xu ${ }^{3}$, Hongwei Ding ${ }^{2}$ \\ ${ }^{1}$ Institute of Chinese Minority Languages and Literature, Minzu University of China \\ ${ }^{2}$ Institute of Cross Language Processing Center, Shanghai Jiao Tong University \\ ${ }^{3}$ Department of Speech, Hearing and Phonetic Sciences, University College London \\ https://doi.org/10.36505/ExLing-2017/08/0030/000332
}

\begin{abstract}
The present study investigated three northern Wu dialects: Wuxi, Suzhou, and Ningbo. It is found that, in all three dialects, focus is encoded by increasing the maximum $\mathrm{F}_{0}$ and duration of focused words, and lowering and compressing the $\mathrm{F}_{0}$ and pitch range of post-focus words. These results are consistent with previous findings about $\mathrm{Wu}$ dialect in Shanghai. Northern $\mathrm{Wu}$ dialects therefore seem to encode focus in a similar way to Beijing Mandarin, but different from many languages/dialects spoken in southern China. This finding, together with evidence from gene studies and migration history of $\mathrm{Wu}$ areas, provide further support for the inheritance hypothesis of PFC, according to which all languages with PFC are descendants of a common proto-language in the Middle East.
\end{abstract}

Key words: Wu dialect, prosody, focus, post-focus-compression (PFC)

\section{Introduction}

Focus is the highlighting of part of a sentence against the rest of the sentence, motivated by discourse context. A focused word is generally realized with increased $\mathrm{F}_{0}$, duration and intensity. $\mathrm{Xu}$ et al. (2012) found that, for the post-focus part, two different patterns have been reported across languages: with post-focus lowering and compression of $\mathrm{F}_{0}$ and intensity (PFC), or without PFC. PFC has been found that in many languages/dialects spoken in northern and middle China, e.g., Uygur, Ando Tibetan, Lasha Tibetan, Beijing Mandarin, Nanchang dialect, etc., but is absent in many southern languages/dialects, e.g., Taiwanese , Deang, Yi and Wa, etc, as summarized in $\mathrm{Xu}$ et al. (2012). They proposed an inheritance hypothesis, according to which PFC is inherited from a common proto-language shared by all languages with the feature.

$\mathrm{Wu}$ area in China is especially interesting for testing the inheritance hypothesis because (1) there are contrary results on the presence of PFC in Wu dialects; (2) the formation of Wu dialects involves influences from both PFC and non-PFC languages in its history. According to Pan (2009), among many others, although $\mathrm{Wu}$ dialects are heavily influenced by Kam-Tai languages back to Chunqiu Dynasties (770-476 B.C.), modern Wu dialects (possibly formed in Sunwu era (220-280)) are

ExLing 2017: Proceedings of 8th Tutorial and Research Workshop on Experimental Linguistics, 19-22 June, Heraklion, Crete, Greece 
mainly spoken by people who immigrated from the north. Wen et al. (2004) showed that the contribution of $\mathrm{Y}$ chromosome and mitochondrial DNA (mtDNA) from northern $\mathrm{Han}$ is about $80 \%$ in the $\mathrm{Wu}$ population (Jiangsu and Shanghai). Thus, the inheritance hypothesis would predict that $\mathrm{Wu}$ languages have PFC just like most northern Mandarin dialects. The current study is an investigation of prosodic focus in the $\mathrm{Wu}$ dialects of Wuxi, Suzhou, and Ningbo.

\section{Method}

Corrective focus was elicited by a preceding sentence, in a structure as "It is not my brother. My sister poured noodle soup". In each dialect, two declarative sentences were constructed to examine focus in four conditions: initial (IF), medial (MF), final (FF) and neutral (NF).

There were 6 Wuxi speakers ( 4 F, 2 M, age 24-52), 8 Suzhou speakers (4F, 4M, age 45-63), and 8 Ningbo speakers (5F, 3M, age 24-45).

The recording was digitized into a computer (Lenovo Z475) by $16 \mathrm{Bit} / 44.1 \mathrm{k}$ using audio sound (Yamaha Steinberg CI2) and a condenser microphone (SHUER Beta 53). Each speaker recorded all the 8 sentences three times in separately randomized blocks.

The data extraction was done with the Praat script ProsodyPro (Xu, 2013), which generated 10 normalized F0 points, maximum F0, and duration for each manually segmented syllable. The F0 values were converted from $\mathrm{Hz}$ to semitones (st) using the following formula:

$$
f_{\text {st }}=12 \log _{2}\left(F_{0}\right)
$$

\section{Results}

\section{$\mathbf{F}_{\mathbf{0}}$}

Fig. 1 displays the time-normalized $\mathrm{F}_{0}$ contours of one sentence in each dialect, with four focus conditions overlaid in one figure. The other sentences showed the same pattern.

It can be seen from Fig. 1 that, in all three dialects, focus raises the maximum $\mathrm{F}_{0}$ of the on-focus word, and compresses the maximum $\mathrm{F}_{0}$ of the post-focus part as compared to the neutral focus condition, but leaves the pre-focus part largely intact.

Table 1 shows mean maximum F0 of the three target words averaged across the two sentences in the three dialects, broken down by the four focus conditions. Two-way repeated measures ANOVA with word position and focus condition as the independent variables were carried out for each dialect. Focus has significant effects in all the three dialects (Wuxi: $F(3,15)=6.45^{*}$, Suzhou: $F(3,21)=14.73^{* *}$, Ningbo: $F(3$, $21)=14.31 * * *)$. 


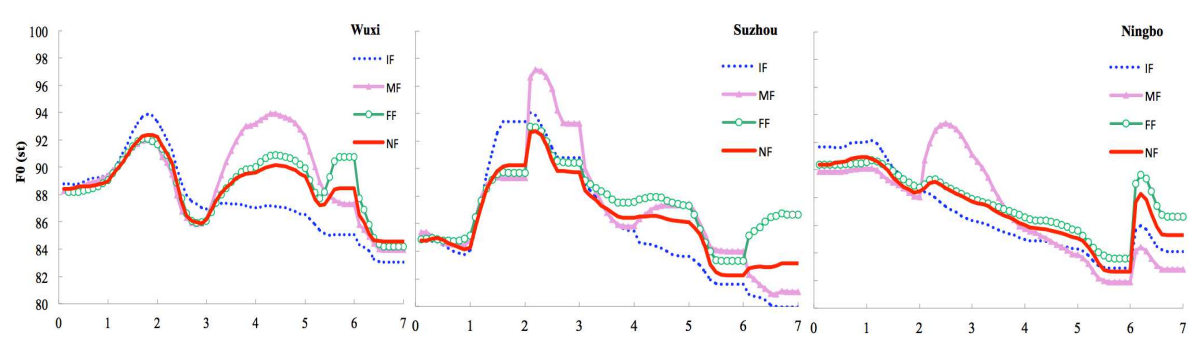

Figure 1. $\mathrm{F}_{0}$ contours of one sentence in the three $\mathrm{Wu}$ dialects.

\begin{tabular}{|c|c|c|c|c|c|}
\hline \hline & Word & IF & MF & FF & NF \\
\hline \multirow{3}{*}{ Wuxi } & W1 & 94.5 & 92.6 & 92.3 & 92.7 \\
& W2 & 92.1 & 92.7 & 91.5 & 91.7 \\
& W3 & 86.6 & 91.3 & 90.3 & 89.2 \\
\hline \multirow{3}{*}{ Suzhou } & W1 & 95.2 & 94.4 & 92.5 & 92.9 \\
& W2 & 91.1 & 95.3 & 92.2 & 91.3 \\
& W3 & 83.6 & 86.8 & 90.7 & 87.1 \\
\hline \multirow{3}{*}{ Ningbo } & W1 & 93.9 & 92.0 & 92.5 & 92.6 \\
& W2 & 87.7 & 92.6 & 89.8 & 89.7 \\
& W3 & 85.9 & 85.6 & 89.8 & 88.4 \\
\hline \hline
\end{tabular}

Table 1. The Maximum $\mathrm{F}_{0}(\mathrm{st})$ of the three target words.

\section{Word Duration}

Table 2 shows mean duration of the three target words under different focus conditions in each dialect, averaged across three repetitions of the two sentences by all speakers.

\begin{tabular}{|c|c|c|c|c|c|}
\hline \hline & Word & IF & MF & FF & NF \\
\hline Wuxi & W1 & 417 & 375 & 369 & 368 \\
\hline & W2 & 412 & 492 & 432 & 431 \\
\hline & W3 & 440 & 444 & 546 & 485 \\
\hline Suzhou & W1 & 528 & 482 & 451 & 468 \\
\hline & W2 & 390 & 484 & 416 & 416 \\
\hline & W3 & 436 & 450 & 549 & 495 \\
\hline Ningbo & W1 & 337 & 314 & 304 & 310 \\
\hline & W2 & 340 & 374 & 366 & 355 \\
\hline & W3 & 456 & 450 & 496 & 484 \\
\hline \hline
\end{tabular}

Table 2. The word duration (ms) of the three target words. 
It can be seen in Table 2 that on-focus words are lengthened in all three dialects. Pre- and post-focus words do not differ much from the neutral focus words. Two-way repeated measures ANOVAs with focus and word position as two independent variables show that focus has main effect in all the dialects (Wuxi: $F(3,15)=4.7 *$; Suzhou: $F(3,21)=3.46^{* * *}$; Ningbo: $F(3$, 21) $\left.=3.16^{*}\right)$.

\section{Discussion and Conclusions}

This study investigated focus encoding in Wuxi, Suzhou, and Ningbo. It is found that, in all these dialects, focus not only raises the maximum F0 and lengthens the duration of the on-focus word, but also compresses and lowers the F0 of the post-focus words. Prosodic focus in these $\mathrm{Wu}$ dialects thus shows a similar pattern to Shanghai (Chen, 2008; Selkirk \& Shen, 1990). Scholtz (2012) has studied a southern Wu dialect (Wenzhou) and showed PFC applied in the initial focus condition. Overall, the results are consistent with the prediction by the inheritance hypothesis of PFC. Nevertheless, more languages/dialects need to be studied, especially southern $\mathrm{Wu}$ dialects.

\section{Acknowledgements}

This paper was jointly supported by the Program for New Century Excellent Talents in University (NCET-12-0584) to B.W and the Interdisciplinary Program of Shanghai Jiao Tong University (14JCZ03) to H-W.D.

\section{References}

Pan, W.Y. 2009. The Historical Background of the Formation of Wu Dialects（吴语形成的历史背景）. Dialect（方言), 3, 193-203.

Selkirk, E., Shen, T. 1990. Prosodic domains in Shanghai Chinese. In The Phonology-Syntax Connection. S. Inkelas and D. Zec. Chicago: University of Chicago Press pp. 313-37.

Wen, B., Shi, H., Ren, L., Xi, H., Li, K., Zhang, W., . . X Xiao, C. 2004. The origin of Mosuo people as revealed by mtDNA and Y chromosome variation. Science in China Series C: Life Sciences, 47(1), 1-10.

$\mathrm{Xu}$, Y. 2013. ProsodyPro - A tool for large-scale systematic prosody analysis. In Proceedings of Tools and Resources for the Analysis of Speech Prosody (TRASP 2013), Aix-en-Provence, France: 7-10.

$\mathrm{Xu}$, Y., Chen, S.-w., Wang, B. 2012. Prosodic focus with and without post-focus compression (PFC): A typological divide within the same language family? The Linguistic Review, 29, 131-147.

Scholtz, F. 2012. Tone sandhi, prosodic phrasing, and focus marking in Wenzhou Chinese. Ph.D. thesis, Leiden University, the Netherlands. 